1 Lethal effects of high intensity violet 405-nm light on Saccharomyces

2 cerevisiae, Candida albicans and on dormant and germinating spores

\section{of Aspergillus niger}

L.E. Murdoch, K. McKenzie, M. Maclean*, S.J. MacGregor and J.G. Anderson The Robertson Trust Laboratory for Electronic Sterilisation Technologies, University of Strathclyde, 204 George Street, Glasgow, Scotland

* Corresponding author. Mailing address: The Robertson Trust Laboratory for Electronic Sterilisation Technologies, Department of Electronic and Electrical Engineering, University of Strathclyde, Royal College Building, 204 George Street, Glasgow, Scotland, G1 1XW. Phone: +44 (0)141 5482891 Fax: +44 (0)141 552 5398 E-mail: michelle.maclean@strath.ac.uk

Running Title: 405-nm light inactivation of fungi

Keywords: Aspergillus; yeast; 405-nm light; spores; germination; inactivation. 


\section{Abstract}

This study assessed the effects of high intensity violet light on selected yeast and mould fungi. Cell suspensions of Saccharomyces cerevisiae, Candida albicans and dormant and germinating spores (conidia) of the mould Aspergillus niger were exposed to high intensity narrow band violet light with peak output at $405 \mathrm{~nm}$ generated from a light emitting diode (LED) array. All three fungal species were inactivated by the $405 \mathrm{~nm}$ light without a requirement for addition of exogenous photosensitiser chemicals. Of the fungal species tested, S. cerevisiae was most sensitive and dormant conidia of $A$. niger were most resistant to $405 \mathrm{~nm}$ light exposure. Five $\log _{10} \mathrm{CFUml}^{-1}$ reductions of the tested species required exposure doses of $288 \mathrm{~J} \mathrm{~cm}^{-2}$ for $S$. cereviaiae, $576 \mathrm{~J} \mathrm{~cm}^{-2}$ for $C$. albicans and a much higher value of $2.3 \mathrm{~kJ} \mathrm{~cm}^{-2}$ for dormant conidia of $A$. niger. During germination, A. niger conidia became more sensitive to $405 \mathrm{~nm}$ light exposure and sensitivity increased as germination progressed over an 8 hour test period. Light exposure under aerobic and anaerobic conditions, together with results obtained using ascorbic acid as a scavenger of reactive oxygen species, revealed that 405-nm light inactivation in fungi involved an oxygen-dependent mechanism, as previously described in bacteria. The inactivation results achieved with yeast cells and fungal spores together with operational advantages associated with the use of a visible (non UV) light source highlights the potential of 405-nm light for fungal decontamination applications. 


\section{Introduction}

There have been extensive studies carried out on the effects of light on the biology of fungal organisms with numerous reports published on effects on growth, metabolism and differentiation. There has also been considerable interest in the application of light-based technologies, using specific light wavelengths and intensities, for the inactivation and control of problematic fungal organisms. Ultraviolet light (UV) in particular has been extensively studied as an inactivation technology for destruction of pathogenic and spoilage microorganisms including fungi and yeasts (Begum et al. 2009). Although UV-light can be effective, it has limitations as a decontamination technology due to its low penetration into opaque liquids and solids, photodegradation of plastics, and human exposure safety issues (Elmnasser et al., 2007).. These limitations associated with UV-light radiation create potential opportunities for alternative light-based decontamination technologies that are safer to use and cause less degradation of photosensitive materials.

Novel technologies utilising visible wavelengths of light, most notably in the violet/blue region of the electromagnetic spectrum that induce so-called photodynamic inactivation (PDI) of microorganisms have been developed. Traditionally PDI has involved the use of dyes and other exogenous photosensitiser molecules coupled with light exposure to induce inactivation, but more recently natural photosensitiser molecules, particularly porphyrins endogenous within microbial cells have been targeted. Exposure of microorganisms to visible light particularly at wavelengths of $405 \mathrm{~nm}$, has been shown to be effective in inactivating a range of bacteria, including Gram positive and Gram negative bacterial species and antibiotic resistant microorganisms such as Methicillin-resistant Staphylococcus 
aureus, and its use has been suggested for a range of medical and environmental decontamination applications (Guffey \& Wilborn 2006; Enwemeka et al. 2008; Maclean et al. 2008a; Maclean et al. 2009; Maclean et al, 2010; Murdoch et al. 2012, Dai et al, 2012; Dai et al, 2013). However use of 405-nm light for inactivation of fungal organisms and the inactivation process involved has not been previously reported.

In prokaryotes, 405-nm light inactivation has been shown to be an oxygen-dependent process that is thought to involve absorption of the 405-nm light by endogenous porphyrin molecules. Light at 405-nm wavelength excites these molecules and leads to transfer of energy. This induces generation of highly reactive singlet oxygen $\left({ }^{1} \mathrm{O}_{2}\right)$ and other reactive oxygen species (ROS) that cause cellular damage and ultimately cell death (Hamblin \& Hasan, 2004; Maclean et al. 2008b; Lipovsky et al. 2010). Studies into broadband light inactivation of fungi have shown the sites of damage caused by singlet oxygen are typically the plasma membrane and mitochondria (Donnelly et al. 2008). This is likely to be where endogenous porphyrins reside in fungi as singlet oxygen can only diffuse a short distance and has a relatively short life-span $\left(10^{-6} \mathrm{~s}\right)$ (Bertoloni et al. 1987; Dougherty et al. 1998; Kalka et al. 2000).

Fungi in general are considered to be more difficult to inactivate than bacteria by some decontamination procedures because of their larger size and complex structures. UV light studies have shown that fungal spores in particular require higher UV light dosage to achieve the same level of inactivation seen in bacterial cells. A study by Anderson et al. (2000) achieved 7-8- $\log _{10}$ reductions in Escherichia coli, Salmonella enteritidis, and Bacillus cereus cells upon exposure to 1000 pulses of high-UV 
content broadband light, whereas fungal spores from Fusarium culmorum and Aspergillus niger were only inactivated by $3-4-\log _{10}$ after treatment. Fungi are also protected from external ROS-induced damage by a thick cell wall containing components such as chitin, mannan, glucan, and various lipids (Donnelly et al. 2008). In addition to the thick outer wall, fungi have a plasma membrane separated from the cell wall by a periplasmic space. This reduces the diffusion of photodynamic sensitising agents, used in PDI, into cells thereby limiting singlet oxygen cell damage.

The present study sets out to assess the effects of 405-nm light, without the addition of exogenous photosensitisers, on cells of S. cerevisiae and C. albicans and on dormant and germinating conidia of A. niger. These fungal species were chosen due to their recognised significance as test organisms in scientific studies and because of their applied importance in medical, industrial and food mycology (Straus, 2009; Mean et al. 2008; Luksiene et al. 2004; Blacketer et al. 1993). This study also aims to determine whether the 405-nm light inactivation of fungi involves an oxygendependant mechanism as has been established in similar studies on bacterial species.

\section{Materials and Methods}

\subsection{Yeast Preparation}

Saccharomyces cerevisiae MUCL 28749 and Candida albicans MUCL 29903

cultures were obtained from the Mycotheque de l'Universite catholique de Louvain (MUCL) (part of The Belgian Co-ordinated Collections of Microorganisms (BCCM)). Saccharomyces cerevisiae and C. albicans were inoculated into $100 \mathrm{ml}$ Malt Extract Broth and Malt Extract Broth with $0.1 \%$ yeast extract, respectively (Oxoid, UK). After incubation for $18-24$ hours at $30^{\circ} \mathrm{C}$ and $37^{\circ} \mathrm{C}$ for $S$. cerevisiae and C. albicans, 
respectively, broths were centrifuged at $3939 \times \mathrm{g}$ for 10 minutes and the resultant cell pellet was re-suspended in $100 \mathrm{ml}$ phosphate buffered saline (PBS; Oxoid, UK). Yeast suspensions were serially diluted to give an approximate starting population of $\sim 2.0 \times 10^{5} \mathrm{CFU} \mathrm{ml}^{-1}$ for experimental use.

\subsection{Aspergillus niger Spore and Mycelia Preparations}

Aspergillus niger MUCL 38993 was obtained from the Mycotheque de l'Universite catholique de Louvain (MUCL). Aspergillus niger spores were inoculated onto Malt Extract Agar slopes and grown at $30^{\circ} \mathrm{C}$ for five days to produce sufficient conidial production (spore production) for use in experimentation. Slopes were flooded with PBS containing one drop of Tween 80 (Sigma, UK) (added to prevent aggregation of spores) and the end of a plastic L-shaped spreader was used to gently remove spores from the surface of the agar slope. One-ml of the resultant solution was then pipetted into $9 \mathrm{ml}$ of PBS before the spores were counted using an Improved Neubauer haemocytometer (Weber Scientific International, UK) to ascertain the population density of the spore suspension. Spore suspensions were then diluted to $\sim 2.0 \times 10^{5}$ $\mathrm{CFU} \mathrm{ml} l^{-1}$ for experimental use. A. niger mycelia were cultured by inoculating $100 \mu \mathrm{L}$ A. niger spores into $100 \mathrm{~mL}$ Malt Extract Broth and incubating under rotary conditions (120 rpm) for 24 hours at $30^{\circ} \mathrm{C}$. Mycelia were then dispersed by stomaching (Don Whitley Scientific, UK) in PBS.

\subsection{High Intensity 405-nm light source}

High-intensity 405-nm light was produced by an indium-gallium-nitride (InGaN) 99DIE light-emitting diode (LED) array (Opto Diode Corp, USA), with a centre 
wavelength $(\mathrm{CW})$ of around $405 \mathrm{~nm}$ and a bandwidth of $\sim 10 \mathrm{~nm}$ at full-width halfmaximum (FWHM). In effect although the light source produced light of $405 \mathrm{~nm}( \pm 5$ $\mathrm{nm}$ ), this will, for convenience, be referred throughout the text as 405-nm light. The LED array was powered by a DC power supply, and a cooling fan and heat sink were attached to the array to dissipate heat from the source, which also served to minimise heat transfer to the sample throughout treatment. The LED array was mounted in a PVC housing designed to fit a 12-well plate (NUNC, Denmark), with the array positioned directly above one of the central sample wells. Irradiance levels of 40 and $63 \mathrm{~mW} \mathrm{~cm}^{-2}$ at the surface of the fungal suspensions were used for exposure of the yeast and spore samples, respectively, and the applied light dose was calculated as the product of the irradiance $\left(\mathrm{W} \mathrm{cm}^{-2}\right)$ multiplied by the exposure time (s).

\subsection{High-intensity 405-nm Light Inactivation Method}

A $3 \mathrm{ml}$ volume of yeast or fungal spore suspension was pipetted into a sample well giving a liquid depth of approximately $10 \mathrm{~mm}$. A micro-magnetic follower was placed in the well, and the plate placed onto a magnetic stirring plate for continuous agitation of the sample. The LED array was placed directly above the sample well, with a distance of approximately $2 \mathrm{~cm}$ between the light source and the liquid surface, and samples were exposed to increasing durations of high-intensity 405-nm light. Control samples which received no high-intensity 405-nm light exposure were also set-up. Temperature of fungal samples was monitored using a thermocouple to ensure there was no build-up of heat from the LED arrays during this exposure time.

\subsection{Oxygen Dependence Experiments}


Exposure of fungal populations in oxygen-depleted environments was carried out as follows. Fungal suspensions were prepared in an anaerobic cabinet (Don Whitley Scientific, UK) using PBS that had acclimatised in the anaerobic environment for at least an hour before use. $405 \mathrm{~nm}$ light exposure of fungal suspensions was then performed in the anaerobic cabinet with the applied doses used being selected based on the lethal dose required to achieve inactivation of each organism in the aerobic (benchtop) experiments. Anaerobic and aerobic non-exposed controls were also setup for each test sample. To further deplete the levels of available oxygen, exposure in the anaerobic cabinet was repeated with a scavenger of reactive oxygen species added to the fungal suspensions. The scavenger and concentration used was $30 \mathrm{mM}$ ascorbic acid (Sigma Aldrich, Dorset, UK), as used in previous studies (Feuerstein $e t$ al., 2005; Maclean et al., 2008b). Exposure of the organisms in the presence of the scavenger was also performed under aerobic conditions as an experimental control.

\subsection{Effect of $405 \mathrm{~nm}$ light on Germinating Aspergillus Spores}

A. niger spore suspensions were prepared as previously described but resuspended in a chemically-defined germination medium $\left(\mathrm{KH}_{2} \mathrm{PO}_{4} 1.0 \mathrm{~g}, \mathrm{MgSo}_{4} 0.25 \mathrm{~g}, \mathrm{CuSO}_{4}\right.$ $0.2 \mathrm{mg}, \mathrm{FeSO}_{4} 6.0 \mathrm{mg}, \mathrm{ZnSO}_{4} 1.0 \mathrm{mg}, \mathrm{MnCl}_{2} 4.0 \mathrm{mg}, \mathrm{CaCl}_{2} 50 \mathrm{mg}, \mathrm{NH}_{4} \mathrm{SO}_{4} 2.0 \mathrm{~g}$, Sucrose $10.0 \mathrm{~g}$, monosodium glutamate $5.0 \mathrm{~g} \mathrm{l}^{-1}$ ) rather than PBS. This germination medium was used as it was clear and transparent thereby facilitating light exposure experiments without light attenuation complications. Ten millilitre volumes were dispensed into petri dishes and held at $30^{\circ} \mathrm{C}$ in a static incubator (to prevent clumping during germination, an effect that occurs more readily under shaken flask cultivation conditions) for up to 8 hours. At 2-hour intervals, $3 \mathrm{ml}$ volumes of germinating spore suspension were removed and exposed to a dose of $454 \mathrm{Jcm}^{-2} 405 \mathrm{~nm}$ light. Non- 
exposed control samples were set up simultaneously with test samples. Results then allowed comparison of the susceptibility of spores at different stages of germination to that of dormant spore populations.

To investigate whether $405 \mathrm{~nm}$ light exposure had an effect on morphological changes during germination, spores were incubated in germination medium for 6 hours at $30^{\circ} \mathrm{C}$, exposed to $454 \mathrm{Jcm}^{-2} 405 \mathrm{~nm}$ light, and re-incubated for a further 2 hours. Microscopic examination of the light-exposed fungal samples and the nonexposed control samples (which had been incubated for 8 hours with no lightexposure) enabled a visual comparison of whether the light exposure had an effect on morphological changes during germination. For photographic purposes, fungal samples were centrifuged and resuspended in $1 / 10^{\text {th }}$ of the volume to effect cell aggregation and increase the cell density in the field of view.

\subsection{Plating and Enumeration}

S. cerevisiae and C. albicans samples were plated onto Malt Extract Agar, and Malt Extract Agar containing $0.1 \%$ yeast extract, respectively (Oxoid, UK). A WASP 2 spiral plater (Don Whitely Scientific Ltd, UK) was used to plate out samples (50 $\mu \mathrm{l}$ spiral plate and $100 \mu \mathrm{l}$ spread plate samples), with each sample being plated in a minimum of triplicate. Sample plates were then incubated at $30^{\circ} \mathrm{C}$ and $37^{\circ} \mathrm{C}$, respectively, for 18-24 hours before enumeration. Results were recorded as colony forming units per millilitre $\left(\mathrm{CFU} \mathrm{ml}{ }^{-1}\right)$. Samples of $A$. niger were, diluted if required, and manually spread plated (50 $\mu \mathrm{l}$ and $100 \mu \mathrm{l}$ volumes) onto Malt Extract Agar in triplicate and incubated at $30^{\circ} \mathrm{C}$ for five days. Colonies were enumerated and 
recorded as $\mathrm{CFU} \mathrm{ml} \mathrm{ml}^{-1}$. Due to the plating techniques used the detection limit of these experiments is $<10 \mathrm{CFU} \mathrm{ml}^{-1}$.

\subsection{Statistical Analysis}

In the 405-nm light exposure experiments, data points on each figure represent the mean results of two or more independent experiments, with each individual experimental data point being sampled in triplicate at least. Data points also contain the standard deviation and significant differences attained from results. Significant differences were calculated at the $95 \%$ confidence interval using ANOVA (one-way) with MINITAB software release 15 and are highlighted with asterisks (*).

\subsection{Fluorescence Spectrophotometry}

The presence of endogenous porphyrins within the fungal species was determined by fluorescence spectrophotometry. Cell cultures were twice washed with PBS, and the resultant cell pellets were suspended in $1 \mathrm{ml}$ of $0.1 \mathrm{M} \mathrm{NaOH}-1 \%$ sodium dodecyl sulphate (SDS) for 24 hours in the dark. Cell suspensions were then centrifuged and the supernatant was used for fluorescence measurements (RF-5301 PC spectrofluorophotometre; Shimadzu, US). Excitation was carried out at $405 \mathrm{~nm}$ and emission spectra were recorded between 500-800 nm.

\section{Results}

\subsection{High-intensity 405-nm Light Inactivation of Yeasts and Fungal Spores}

Results in Figure 1 demonstrate that $C$. albicans was inactivated by $5-\log _{10} \mathrm{CFU} \mathrm{ml}{ }^{-1}$ upon exposure to $405-\mathrm{nm}$ light at a dose of $576 \mathrm{~J} \mathrm{~cm}^{-2}$. S. cerevisiae showed higher susceptibility, with a $5-\log _{10} \mathrm{CFU} \mathrm{ml} \mathrm{m}^{-1}$ reduction achieved in half the dosage required 
249 for C. albicans $\left(288 \mathrm{~J} \mathrm{~cm}^{-2}\right)$. Inactivation data for A. niger dormant spore suspensions

250 is also shown in Figure 1. When dose levels similar to those used to achieve a 5-

$251 \log _{10} \mathrm{CFUml}^{-1}$ reduction in population of the yeast populations (up to approximately

$252500 \mathrm{Jcm}^{-2}$ ) were used, no reduction of dormant $A$. niger spores was evident.

253 However, with the application of higher doses there was a steady decrease in

254 population, with a $5-\log _{10} \mathrm{CFU} \mathrm{ml}{ }^{-1}$ reduction of $A$. niger spores achieved with a dose

255 of $2.3 \mathrm{~kJ} \mathrm{~cm}^{-2}$ - almost five times the dose required for the same 5 - $\log _{10}$ reduction of

256 the yeast cells. Control samples for both the yeast and fungal spore populations did

257 not significantly change over the duration of the experiment. No significant increase

258 in the temperature of the exposed fungal samples was recorded during light exposure,

259 with the maximum temperature recorded being $29^{\circ} \mathrm{C}\left( \pm 1{ }^{\circ} \mathrm{C}\right)$ after application of the

260 highest dose $\left(2.3 \mathrm{~kJ} \mathrm{~cm}^{-2}\right)$.

\section{Figure 1}

\subsection{Oxygen Dependence Experiments}

Fungal samples were light-exposed in an anaerobic cabinet to doses which achieved a

5- $\log _{10} \mathrm{CFUml}^{-1}$ reduction under normal aerobic atmospheric conditions (which were $288 \mathrm{~J} \mathrm{~cm}^{-2}$ for $S$. cerevisiae; $576 \mathrm{~J} \mathrm{~cm}^{-2}$ for C. albicans; $2.3 \mathrm{~kJ} \mathrm{~cm}^{-2}$ for A. niger).

Results, which are shown in Table 1, demonstrate that the normally 'lethal' 405-nm light dose had no significant effect on A. niger spores exposed in the absence of oxygen, with only $0.1-\log _{10} \mathrm{CFU} \mathrm{ml} \mathrm{l}^{-1}$ inactivated. Inactivation of $C$. albicans was

271 also reduced in the anaerobic environment with only $1.8-\log _{10} \mathrm{CFU} \mathrm{ml}{ }^{-1}$ inactivated.

272 Inactivation of S. cerevisiae did not appear to be as oxygen-dependent as the other

273 two species, as a reduction of 4.4- $\log _{10} \mathrm{CFU} \mathrm{ml}{ }^{-1}$ was achieved when light-exposed in 
the anaerobic environment. Although the effect was less pronounced with S. cerevisiae, statistical analysis confirmed that for all three fungal species tested there was a statistically significant difference in the population reductions when exposed in the aerobic versus the anaerobic environments.

In order to further examine oxygen-dependence in the test yeasts, ascorbic acid, a scavenger of reactive oxygen species, was added to the cell suspensions, which were held in the anaerobic cabinet, as an additional measure to minimise the residual dissolved oxygen in the samples. As a definitive oxygen-dependent effect had been found with A. niger, no further scavenger testing was carried out on this organism. Results of exposing scavenger-supplemented suspensions of $C$. albicans and $S$. cerevisiae to 405-nm light under anaerobic and aerobic conditions are shown in Table 1. Ascorbic acid was effective at inhibiting inactivation with C. albicans, with no significant inactivation of $C$. albicans occurring when exposed anaerobically in the presence of ascorbic acid. This was significantly different to the $1.8 \log _{10}$ reduction achieved when exposed anaerobically in the absence of ascorbic acid. Presence of ascorbic acid also reduced the level of inactivation of $S$. cerevisiae, with a $2.3 \log _{10}$ difference between anaerobically-exposed cells in the presence and absence of the scavenger. Exposure of C. albicans and S. cerevisiae in the presence of ascorbic acid under aerobic conditions resulted in 3.2 and $2.7 \log _{10}$ reductions, respectively; significantly less than the $5 \log _{10}$ reductions achieved when exposed under the same conditions in the absence of the scavenger. Overall, although differences between the organisms were noted, results in Table 1 demonstrate that for both organisms, most inactivation was achieved when exposed in aerobic conditions in the absence of the 
scavenger, and the most effective reduction of microbial inactivation was achieved using the scavenger in the anaerobic environment.

\section{Table 1}

\subsection{Effect of $405 \mathrm{~nm}$ Light on Germinating Spores}

Results in Figure 1 demonstrated the high $405 \mathrm{~nm}$ light doses required to cause inactivation of dormant $A$. niger spores. Experiments were carried out to determine whether germinating spores were more susceptible to $405 \mathrm{~nm}$ light. Figure 2 demonstrates the results achieved when A. niger spores were incubated in a germination medium prior to light exposure using a dose of $454 \mathrm{~J} \mathrm{~cm}^{-2}$. A significant reduction in the CFU count of the spore suspension was achieved when the spores were light-exposed after a 2-hour incubation period in the germination medium.

When the period in germination medium, prior to light exposure, was extended, reductions progressively increased from 0.8 to $2.5 \log _{10} \mathrm{CFU} \mathrm{ml}{ }^{-1}$ reduction over the 8 hour test period. No significant change in CFU count was recorded with dormant conidia exposed to the same dose of $454 \mathrm{~J} \mathrm{~cm}^{2}$; therefore demonstrating that once germination of conidia is initiated, conidia have an increased sensitivity to $405 \mathrm{~nm}$ light, with their sensitivity increasing as germination progresses.

\section{Figure 2}

The effect of $405 \mathrm{~nm}$ light exposure on morphological changes during the germination process was also investigated. Microscopic examination of conidia after 2, 4, 6 and 8 hours incubation in germination medium showed that the conidia underwent the typical germination process previously described for $A$. niger conidia 
323 (Anderson and Smith, 1971). Dormant conidia (3.5 $\mu \mathrm{m}$. mean diameter), which

324 possessed a rough dark pigmented spore coat (Fig 3a), increased in diameter by a

325 process of both imbibitional and spherical growth swelling to 6.5 to $7.0 \mu \mathrm{m}$. before

326 germ-tube outgrowth. The majority of conidia produced one or occasionally two germ

327 tubes after 6 hours. The effect of $405 \mathrm{~nm}$ light exposure when applied during the

328 germination process can be clearly seen in Figures 3b-c which illustrates the

329 significant difference in the extent of germination between light-exposed and non-

330 exposed spores.

331

332

333

334

335

\section{Figure 3}

\subsection{Fluorescence Spectrophotometry}

Figure 4 shows the fluorescence emission spectra of suspension preparations of the three fungal species dissolved in $\mathrm{NaOH}-\mathrm{SDS}$. Excitation of the cell supernatants at $405 \mathrm{~nm}$ displayed emission peaks at 608 and $611 \mathrm{~nm}$ for $C$. albicans and S. cerevisiae, respectively. No peaks were observed for $A$. niger spores when excited under the same conditions, however analysis carried out using A. niger mycelia demonstrated a peak at $607 \mathrm{~nm}$.

\section{Figure 4}

\section{Discussion}

The results of this study have demonstrated that fungal organisms can be inactivated by exposure to high intensity light from within the visible spectrum and specifically violet light of wavelength $405 \mathrm{~nm}( \pm 5 \mathrm{~nm})$. Of the two yeast species tested, 
S. cerevisiae was more sensitive to 405-nm light exposure than $C$. albicans with the

349 dose levels required to achieve an approximate $5 \log _{10}$ reduction being $\sim 288 \mathrm{~J} \mathrm{~cm}^{-2}$

350 for $S$. cerevisiae and $\sim 576 \mathrm{~J} \mathrm{~cm}^{-2}$ for C. albicans. By contrast, dormant conidia of the

351 filamentous mould A. niger were most resistant, requiring a dose of approximately 2.3

$352 \mathrm{~kJ} \mathrm{~cm}^{-2}$.

353

354 Although the dormant conidia of A. niger required relatively high levels of $405 \mathrm{~nm}$

355 light exposure before inactivation was achieved, results demonstrated that upon

356 germination, the susceptibility of spores significantly increased. Inactivation data

357 over the germination period (Figure 2) showed that an increased susceptibility of the

358 conidia to $405 \mathrm{~nm}$ light was evident after only a 2-hr germination period, indicating

359 that the initial changes undergone by the germinating spores are enough to increase

360 their susceptibility. It can only be speculated whether this increase in susceptibility is

361 related to morphological changes, e.g. increased light penetration associated with

362 stretching or fracture of the dense pigmented spore coat, or to an increased metabolic

363 vulnerability to light induced reactive oxygen species or indeed to a combination of

364 these effects.

365

366 Results of this study also demonstrated that conidial sensitivity increases as the

367 germination process progresses. Microscopic analysis of conidia which had been

368 light-exposed for a period during germination, demonstrated that the $405 \mathrm{~nm}$ light

369 exposure arrested the development of the germinating conidia, with hyphal

370 development appearing stunted compared to non-exposed germinating conidia (Figure

$3713 \mathrm{~b}-\mathrm{c})$, indicating that light exposure during germination can negatively affect both the

372 germ tube and hyphal growth processes. 
374 The resistance to 405 -nm light exhibited by dormant $A$. niger conidia is not surprising

375 given the requirement of these structures to survive periods of exposure to solar

376 radiation during aerial dispersal. Strong resistance to UV exposure has been

377 demonstrated by $F$. culmorum and A. niger spores during inactivation studies

378 involving use of pulsed ultra-violet light radiation (Anderson et. al., 2000). A. niger

379 spores are particularly difficult to inactivate by light, as they possess multi-layered

380 pigmented spore coats containing aspergillin, a black coloured melanin-like

381 compound (Ray \& Eakin, 1975).

382

383 An observation made whilst studying the inactivation effects of 405-nm light on

384 dormant A niger conidia was that some of the spores that survived the light treatment

385 germinated and gave rise to colonies that did not produce spores even after prolonged

386 incubation (Figure 5). A study by Hatakeyama et al. (2007) found that conidiation of

387 Aspergillus oryzae was repressed upon exposure to white light. Interestingly they

388 also found that blue light completely suppressed colony formation, which supports the

389 A. niger results of this study. However, Zhu \& Wang (2005) found that A. niger

390 conidiation actually increased upon exposure to blue light, which is contrary to the

391 aforementioned results. It is likely that variations in the effects of light on conidiation

392 also depend on other factors such as the growth media employed and the light

393 wavelengths and intensities used. In Aspergillus nidulans it has been reported that

394 conidiation can be regulated by light (Ruger-Herreros \& Rodriguez-Romero, 2011)

395 and toxin production and morphogenesis can be controlled by interactions between

396 light and glucose regulation (Atoui et. al., 2010). 
400 Light from the red and blue regions of the spectrum have also been shown to have 401 important effects on spore germination, hyphal growth and mycotoxin formation in 402 the food relevant Aspergillus and Penicillium fungi (Schmidt-Heydt et. al., 2011). At 403 the molecular level there has been considerable interest in light-mediated regulation in 404 fungi which is primarily conferred by blue-light receptors referred to as WHITE

405 COLLARs which are responsible for both light-dependent and -independent 406 processes such as induction of sporulation, carotenoid biosynthesis and circadian 407 rhythms (Corrochano, 2007). Whether or not blue light receptors are involved in any of the morphogenetic or inactivation responses reported in the current study is outwith the scope of this investigation,

Regarding the susceptibility of the fungi to 405-nm light inactivation, it is interesting to compare the results of the current study to those of previously published work on

413 bacteria. The prokaryotic bacteria also exhibit considerable variability in susceptibility with values, to achieve similar $5-\log _{10}$ order reductions, as low as 18 $\mathrm{J} \mathrm{cm}^{-2}$ with Campylobacter jejuni (Murdoch et al., 2010) but most typically around 50 - $300 \mathrm{~J} \mathrm{~cm}^{-2}$, with Gram positive species being generally more susceptible than Gram 417 negatives (Maclean et al., 2009). Comparison with the values for fungi reported in the current study indicates that fungal organisms may be somewhat more resistant to 405nm light than bacteria. Interestingly it has been previously observed that $C$. albicans, 420 and other yeasts, are slightly more difficult to kill by photodynamic antimicrobial 421 chemotherapy (PACT) than Gram positive bacterial cells, thereby necessitating higher 422 drug and light doses (Zeina et. al., 2002). This has been attributed to the presence of a 
nuclear membrane in the yeasts, the greater cell size and the reduced number of targets for singlet oxygen per unit volume of cell (Donnelly et. al., 2008).

It is also interesting that the relatively high levels of $405 \mathrm{~nm}$ light, as reported in the current study, required to inactivate dormant $A$. niger spores are not dissimilar to those required for the inactivation of bacterial endospores. We report here that a 5-log order reduction of dormant conidia of $A$. niger required a dose of $2.3 \mathrm{~kJ} \mathrm{~cm}^{-2}$ whereas Maclean et al (2012) reported that approximately $2 \mathrm{~kJ} \mathrm{~cm}^{-2}$ was required to achieve an approx 4-log order reduction of Bacillus species endospores.

Due to the irradiance output of the light sources used in this study, inactivation of the fungal organisms required relatively long exposure periods, i.e. 2 and 4 hours at an irradiance of $40 \mathrm{~mW} \mathrm{~cm}{ }^{-2}$ for $S$. cerevisiae and C. albicans, respectively, and 10 hours at $63 \mathrm{~mW} \mathrm{~cm}{ }^{-2}$ for fungal spores (for $5-\log _{10}$ reduction). Microbial inactivation by $405 \mathrm{~nm}$ light exposure has been found to be dose-dependent (Murdoch et al, 2012), and in applications where more rapid fungal inactivation is desirable then use of a much higher power light source would significantly reduce the exposure times required for effective treatment.

It is considered highly likely that the inactivation mechanism initiated within the fungal species tested involves the photoexcitation of free porphyrins within the exposed cells, as is indicated for bacterial inactivation (Dai et al, 2012). Fluorescence spectrophotometry was used to confirm the presence of porphyrins within the test organisms. The fluorescence emission spectra of the three species indicated similar 
peaks in the region $605-615 \mathrm{~nm}$, and although the results are qualitative, there was distinct differences in the intensity of the detected peaks. These peaks indicate that the three test species are likely to contain the same predominant intracellular freeporphyrin, possibly coproporphyrin, due to the similarity in its emission peaks when excited at $405 \mathrm{~nm}$ (Dai et al, 2013; Hamblin et al, 2005), however further analysis would be required to provide a full identification.

Most probably due to limitations of the extraction methodology employed, analysis of A. niger spores produced no detectable porphyrin excitation peaks. This may well have been due to the persistence of some of the dark spore pigment extract in the supernatant thereby masking the fluorescence detection of porphyrins. For this reason A. niger mycelia was used as an extraction source to allow a comparative analysis with the yeast species. Exposure of A. niger mycelia to $405 \mathrm{~nm}$ light showed that complete inactivation of a $3.5 \log _{10} \mathrm{CFU}$ population of dispersed mycelia was achieved upon exposure to a dose of $1080 \mathrm{~J} \mathrm{~cm}^{-2}$, a result which demonstrated the increased susceptibility of mycelia compared to the resilient spores.

Comparing the fluorescence emission and the inactivation rates of the three species, it is interesting to note that $S$. cerevisiae, which was the most susceptible to inactivation, had the greatest intensity of intracellular porphyrin, and A. niger, which was least susceptible, had the lowest. This suggests that the intracellular levels of the detected porphyrin may have a direct influence on the susceptibility of the organisms.

\section{The current study has also demonstrated that oxygen plays an important role in the} 405-nm inactivation mechanism in fungi, further supporting the hypothesis that 
inactivation involves the photoexcitation of endogenous porphyrins - an oxygendependent reaction (Hamblin \& Hasan, 2004). The use of anaerobic exposures and a ROS scavenger demonstrated that reducing the oxygen and ROS significantly decreased, or in some cases completely inhibited, 405-nm light induced inactivation. The use of ascorbic acid as the scavenger also provided some information on the nature of the ROS important in the 405-nm light inactivation mechanism. Ascorbic acid can be taken up by the cell and act as a singlet oxygen quencher and radical scavenger (Granot et al, 2003; Maclean et al, 2008b), thus indicating that singlet oxygen and other radicals are key contributors to the inactivation process.

Inactivation of $A$. niger spores was almost completely inhibited when exposed within an anaerobic environment. The yeasts, particularly $S$. cerevisiae, still displayed a degree of inactivation when exposed under anaerobic conditions, which was then further reduced when combined with the scavenger, however a notable level of S. cerevisiae inactivation (2- $\log _{10} \mathrm{CFU} \mathrm{ml} \mathrm{l}^{-1}$ ) was still observed: thus indicating either the increased susceptibility of the organism, or the potential involvement of other factors in the inactivation mechanism for this organism. Although fluorescence spectroscopy results demonstrated that there were similarities in the likely porphyrin content of the three organisms, albeit at different levels, it is unlikely that this is the sole reason for the differences between their inactivation kinetics, and it is possible that additional chromophores contribute to the inactivation of these eukaryotic cells, however elucidation of this was out-with the scope of this study.

Other groups studying the effect of light on yeasts have obtained results that are compatible to those reported here. Fraiken et al. (1996) investigated the use of visible 
light for the inactivation of three strains of S. cerevisiae and C. guillermondii, via a "porphyrin-type compound" bound to the plasma membranes of the yeasts. Upon exposure to the 400-600 nm light, reductions in cell numbers of $93-95 \%$ in $C$. guillermondii and $S$. cerevisiae were achieved $\left(10^{6} \mathrm{CFU} \mathrm{ml}^{-1}\right.$ starting population). They also noted inactivation was greatly reduced when yeasts were exposed to visible light under anoxic conditions and hypothesised that inactivation in these yeast species was most likely driven by singlet oxygen damage. Therefore the results of this current study correlate well with the previous findings that blue light inactivation of microorganisms is an oxygen-dependent process (Fraiken et al., 1996; Feuerstein et al., 2005; Maclean et al., 2008b).

The use of light to control fungal and other spoilage microorganisms, has traditionally involved the use of light from the UV region of the spectrum which is much more biocidal than 405-nm light. A study by Begum et al. (2009) treated different fungi including A. niger using a UV-C lamp emitting light at $254 \mathrm{~nm}$. They found that after an exposure time of 180 seconds, a $2-\log _{10}$ reduction in $A$. niger spores was observed at a total dose of around $0.5 \mathrm{~J} \mathrm{~cm}^{-2}$. Anderson et al. (2000) studied the effects of pulsed UV-light (PUV) on Fusarium culmorum and A. niger. They exposed agar plates seeded with fungi to light pulses produced by a Xenon flashlamp with high UV content resulting in around 3 or $4-\log _{10}$ reductions in A. niger and F. culmorum respectively after 1000 pulses.

Although UV-light is highly biocidal there are problems with the application of this technology including inactivation tailing effects, poor penetrability, and photoreactivation responses with sub-lethally damaged populations. Also human 
health exposure concerns and problems associated with UV-light damage to photosensitive materials are disadvantages associated with the use of UV light as a decontamination technology (Elmnasser et al.2007). Because of the ongoing requirements to control pathogenic and spoilage fungi and because of limitations associated with the use of other decontamination technologies, potential applications may be found for the use of 405 -nm light for the inactivation of problematic yeast and mould fungi.

\section{Conclusions}

High-intensity 405-nm light has been successfully applied for the inactivation S. cerevisiae, C. albicans, and dormant and germinating spores of A. niger, in the presence of oxygen but without the requirement of exogenous photosensitiser molecules. Results indicate that in fungi, 405-nm light inactivation involves a violet light induced, oxygen dependent mechanism similar to that previously described in bacteria. Although 405-nm light is less fungicidal than UV-light, 405-nm light offers potential operational advantages over UV-light radiation, and development of higher intensity systems could find potential applications for surface, air or equipment decontamination in the medical, pharmaceutical and food industries or in any other setting in which reduction of fungal contamination is desirable or essential.

\section{Acknowledgements}

LEM and KM would like to thank the Engineering and Physical Sciences Research Council for (EPSRC) for support through Doctoral Training Grants (Awarded in 2007 and 2010). Thanks to D. Currie and S. Moorhead for mycological technical support, and K. Henderson and M. H. Grant for access and technical support with the 
547 fluorescence spectrophotometry. All authors would also like to thank The Robertson

548 Trust for their funding support.

549

550

$551 \quad$ References

552 Anderson JG, Smith JE, 1971. The production of conidiophores and conidia by newly

553 germinated conidia of Aspergillus niger (microcycle conidiation). Journal of General

554 Microbiology, 69: 185-197.

555

556 Anderson JG, Rowan NJ, MacGregor SJ, Fouracre RA, Farish O, 2000. Inactivation

557 of food-borne enteropathogenic bacteria and spoilage fungi using pulsed-light. IEEE

558 Transactions on Plasma Science 28(1): 83-88.

559

560 Atoui, A, Kastner, C, Larey, CM, Thokala, R, Etxebeste, O, Espeso, EA, Fischer, R,

561 Calvo AM, 2010. Cross-talk between light and glucose regulation controls toxin

562 production and morphogenesis in Aspergillus nidulans. Fungal Genetics and Biology

563 47: 962-972.

564

565 Begum M, Hocking AD, Miskelly D, 2009. Inactivation of food spoilage fungi by

566 ultra violet (UVC) irradiation. International Journal of Food Microbiology 129: 74-

56777.

568

569 Bertoloni G, Zambotto F, Conventi L, Reddi E, Jori G, 1987. Role of specific cellular

570 targets in the hematoporphyrin-sensitized photoinactivation of microbial cells.

571 Photochemistry and Photobiology 46(5): 695-698. 
573 Blacketer MJ, Koehler CM, Coats SG, Myers AM, Madaule P, 1993. Regulation of

574 dimorphism in Saccharomyces cerevisiae: involvement of the novel protein kinase

575 homolog Elm1p and protein phosphatase 2A. Molecular and Cellular Biology 13(9):

$576 \quad 5567-5581$.

577

578 Corrochana, LM, 2007. Fungal photoreceptors: sensory molecules for fungal

579 development and behaviour. Photochemical \& Photobiological Sciences 6: 725-736.

580

581 Dai T, Gupta A, Murray CK, Vrahas MS, Tegos GP, Hamblin MR, 2012. Blue light

582 for infectious diseases: Propionibacterium acnes, Helicobacter pylori, and beyond?

583 Drug Resistance Updates 15: 223-236.

584

585 Dai T, Gupta A, Huang Y, Yin R, Murray CK, Vrahas MS, Sherwood ME, Tegos GP, 586 Hamblin MR, 2013. Blue Light Rescues Mice from Potentially Fatal Pseudomonas

587 aeruginosa Burn Infection: Efficacy, Safety, and Mechanism of Action. Antimicrobial 588 Agents and Chemotherapy 57(3): 1238-1245.

589

590 Donnelly RF, McCarron PA, Tunney MM, 2008. Antifungal photodynamic therapy.

591 Microbiological Research 163: 1-12.

592

593 Dougherty TJ, Gomer CG, Henderson BW, Jori G, Kessel D, Korbelik M, Moan J, 594 Peng Q, 1998. Photodynamic therapy. Journal of the National Cancer Institute 90:

595 889-905.

596 
600

601 Enwemeka CS, Williams D, Hollosi S, Yens D, Enwemeka SK, 2008. Visible 405 nm

602 SLD light photo-destroys Methicillin-resistant Staphylococcus aureus (MRSA) In

603 Vitro. Lasers in Surgery and Medicine 40(10):734-737.

604

605 Feuerstein O, Ginsburg I, Dayan E, Veler D, Weiss EI, 2005. Mechanism of visible

606 light phototoxicity on Porphyromonas gingivalis and Fusobacterium nucleatum.

607 Journal of Photochemistry and Photobiology 81(5): 1186-1189.

608

609 Fraiken GYa, Strakhovskaya MG, Rubin AB, 1996. The role of membrane-bound

610 porphyrin-type compound and endogenous sensitizer in photodynamic damage to

611 yeast plasma membranes. Journal of Photochemistry and Photobiology B 34: 129-

$612 \quad 135$.

613

614 Granot D, Levine A, Dor-Hefetz E, 2003. Sugar-induced apoptosis in yeast cells.

$615 \quad$ FEMS Yeast Research 4: 7-13.

616

617 Guffey JS, Wilborn J, 2006. In Vitro bactericidal effects of 405-nm and 470-nm blue

618 light. Photomedicine and Laser Surgery 24(6): 684-688.

619 
623

626

627

628

629

630

631

632

633

634

635

636

637

638

639

640

641

642

643

644

Hamblin MR, Viveiros J, Yang C, Ahmadi A, Ganz RA, Tolkoff MJ, 2005.

Helicobacter pylori accumulates photoactive porphyrins and is killed by visible light. Antimicrobial Agents and Chemotherapy 49(7): 2822-2827.

Hamblin MR, Hasan T, 2004. Photodynamic therapy: a new antimicrobial approach to infectious disease? Photochemical \& Photobiological Sciences 3: 436-450.

Hatakeyama R, Nakahama T, Higuchi Y, Kitamoto K, 2007. Light represses conidiation in koji mold Aspergillus oryzae. Bioscience Biotechnology and Biochemistry 71(8): 1844-1849.

Kalka K, Merk H, Mukhtar H, 2000. Photodynamic therapy in dermatology. Journal of the American Academy of Dermatology 42: 389-413.

Lipovsky A, Nitzan Y, Gedanken A, Lubart R, 2010. Visible light-induced killing of bacteria as a function of wavelength: implication for wound healing. Lasers in Surgery and Medicine 42: 467-472.

Luksiene Z, Peciulyte D, Lugauskas A, 2004. Inactivation of fungi in vitro by photosensitization: preliminary results. Annals of Agricultural and Environmental Medicine 11: 215-220.

Maclean M, Murdoch LE, MacGregor SJ, Anderson JG, 2013. Sporicidal effects of high-intensity $405 \mathrm{~nm}$ visible light on endospore-forming bacteria. Photochemistry and Photobiology 89(1); 120-126. 
646 Maclean M, MacGregor SJ, Anderson JG, Woolsey GA, Coia JE, Hamilton K,

647 Taggart I, Watson SB, Thakker B, Gettinby G, 2010. Environmental decontamination

648 of a hospital isolation room using high-intensity narrow-spectrum light. Journal of

649 Hospital Infection 76: 247-251.

650

651 Maclean M, MacGregor SJ, Anderson JG, Woolsey GA, 2009. Inactivation of

652 bacterial pathogens following exposure to light from a 405-nm LED array. Applied

653 and Environmental Microbiology. 75(7):1932-1937.

654

655 Maclean M, MacGregor SJ, Anderson JG, Woolsey GA, 2008a. High-intensity

656 narrow-spectrum light inactivation and wavelength sensitivity of Staphylococcus

657 aureus. FEMS Microbiology Letters. 285(2): 227-232.

658

659 Maclean M, MacGregor SJ, Anderson JG, Woolsey GA, 2008b. The role of oxygen in 660 the visible-light inactivation of Staphylococcus aureus. Journal of Photochemistry 661 and Photobiology B. 90(3):180-184.

662

663 Méan M, Marchetti O, Calandra T, 2008. Bench-to-bedside review: Candida

664 infections in the intensive care unit. Critical Care 12(1): 204.

665

666 Murdoch LE, Maclean M, MacGregor SJ, Anderson JG, 2010. Inactivation of

667 Campylobacter jejuni by exposure to high-intensity 405-nm visible light. Foodborne

668 Pathogens and Disease 7(10): 1211-1216.

669 
670

671

672

673

674

675

676

677

678

679

680

681

682

683

684

685

686

687

688

689

690

691

692

693

694

Murdoch LE, Maclean M, Endarko, MacGregor SJ, Anderson JG, 2012. Bactericidal

effects of $405 \mathrm{~nm}$ light exposure demonstrated by inactivation of Escherichia,

Salmonella, Shigella, Listeria, and Mycobacterium species in liquid suspensions and on exposed surfaces. The Scientific World Journal Article ID 137805, 8 pages.

Ray, AC, Eakin, RE, 1975. Studies on the biosynthesis of aspergillin by Aspergillus niger. Applied Microbiology 30: 909-915.

Ruger-Herreros, C, Rodriguez-Romero, J, 2011. Regulation of conidiation in Aspergillus nidulans. Genetics 188(4): 809-822.

Schmidt-Heydt, M, Rufer, C, Raupp, F, Bruchmann, A, Perrone, G, Geisen, R, 2011. Influence of light on food relevant fungi with emphasis on ochratoxin producing species. International Journal of Food Microbiology 145: 229-237.

Straus DC, 2009. Molds, mycotoxins, and sick building syndrome. Toxicology and Industrial Health 25(9-10): 617-635.

Zeina, B, Greenman, J, Corry, D, Purcell, WM, 2002, Cytotoxic effects of antimicrobial photodynamic therapy on keratinocytes in vitro. British Journal of Dermatology 146: 568-573.

Zhu GC, Wang XJ, 2005. Effect of blue light on conidiation development and glucoamylase enhancement in Aspergillus niger. Wei Sheng Wu Хие Bao 45(2): 2758. 
701

702

714 Figure 1. Inactivation of S. cerevisiae, C. albicans and dormant A. niger spores in 715 liquid suspension, by exposure to 405-nm light. Asterisks (*) represent light-exposed 716 populations which were significantly different to non-exposed control populations $717(\mathrm{P} \leq 0.05)$. No significant changes were observed in the control samples throughout 718 the experiment. 
731

732

Figure 2. Demonstration of the increasing susceptibility of $A$. niger conidia to $405 \mathrm{~nm}$ light when exposed at various stages of germination. Conidia were exposed to a dose of $454 \mathrm{~J} \mathrm{~cm}^{-2}$. Data for the exposure of dormant conidia suspended in PBS is included for reference. Asterisks (*) represent light-exposed populations which were significantly different to non-exposed control populations $(\mathrm{P} \leq 0.05)$.

Figure 3. Microscopic visualisation of (a) dormant conidia, (b) conidia exposed to $405 \mathrm{~nm}$ light $\left(454 \mathrm{Jcm}^{-2}\right)$ after a 6-hr germination period followed by a further 2 -hr post-exposure germination period, showing abnormal germination, and (c) nonexposed conidia after an equivalent 8-hr germination period, showing normal germination. Cells were viewed under $\times 400$ magnification.

Figure 4. Fluorescence spectra of Saccharomyces cerevisiae, Candida albicans and Aspergillus niger mycelia. Fluorescence emission spectra were detected from suspension preparations of the three species dissolved in $\mathrm{NaOH}-\mathrm{SDS}$, using an excitation wavelength of $405 \mathrm{~nm}$.

Figure 5. A mixture of sporing and non-sporing A. niger colonies that have developed from spores that were exposed to high doses of $405 \mathrm{~nm}$ light. Light exposed spores were plated onto Malt Extract Agar and incubated for 10 days for colony development. 
747 Table 1. Mean population reductions of fungal test species after exposure to 405-nm

748 light under aerobic and anaerobic conditions. Doses used for exposure were those

749 required to achieve a $5-\log _{10}$ reduction of each organism under aerobic conditions:

$750288 \mathrm{~J} \mathrm{~cm}^{-2}$ for $S$. cerevisiae, $576 \mathrm{~J} \mathrm{~cm}^{-2}$ for C. albicans; $2.3 \mathrm{~kJ} \mathrm{~cm}^{-2}$ for A. niger

751 spores.

752

\begin{tabular}{lcc|cc}
\hline \multirow{2}{*}{ Microorganisms } & \multicolumn{3}{c}{ Mean Reduction in Microbial Numbers $\left(\log _{10} \mathbf{C F U ~ m l}^{-1}\right)$} \\
\cline { 2 - 5 } & \multicolumn{2}{c}{ Light-exposed } & \multicolumn{2}{c}{ Light-exposed + Scavenger } \\
\cline { 2 - 5 } & Aerobic & Anaerobic & Anaerobic & Aerobic \\
\hline C. albicans & $5.02( \pm 0.1)$ & $1.76( \pm 0.3)$ & $0.03( \pm 0.0)$ & $3.25( \pm 1.6)$ \\
S. cerevisiae & $5.18( \pm 0.1)$ & $4.37( \pm 0.8)$ & $2.03( \pm 0.1)$ & $2.72( \pm 0.3)$ \\
A. niger spores & $5.24( \pm 0.2)$ & $0.1( \pm 0.3)$ & N/A & N/A \\
\hline
\end{tabular}

753

754

755

756

757

758

759

760

761

762

763

764

765 


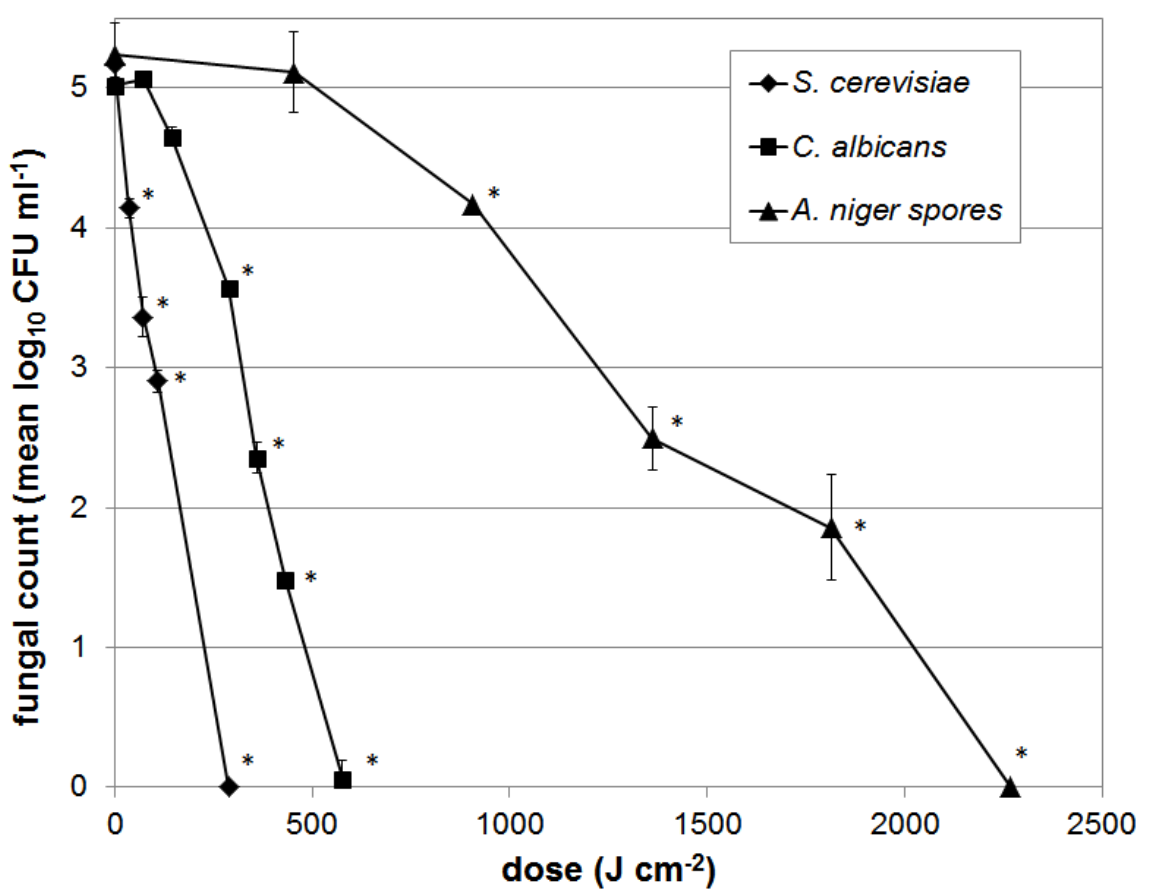

767

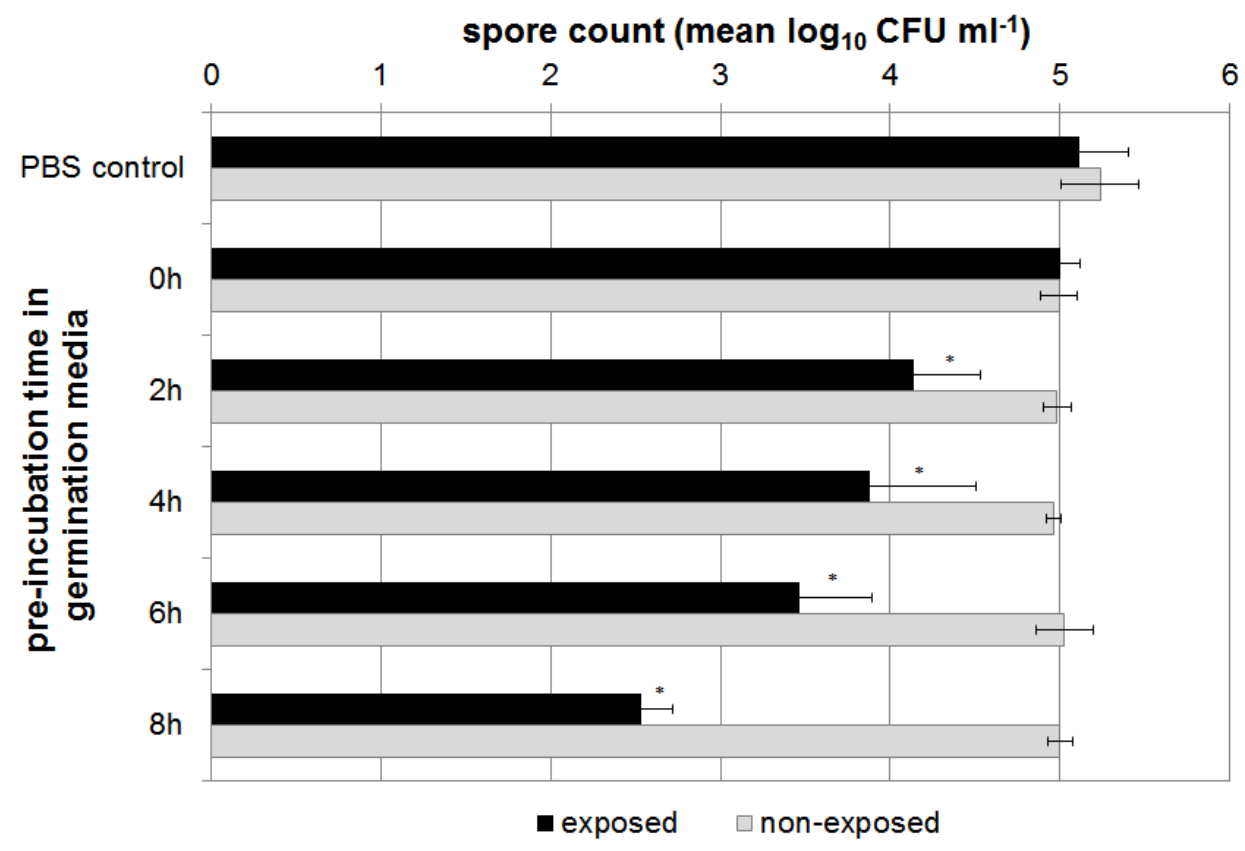

Fig 2 
774

775

776

777

778

779

780

781

782

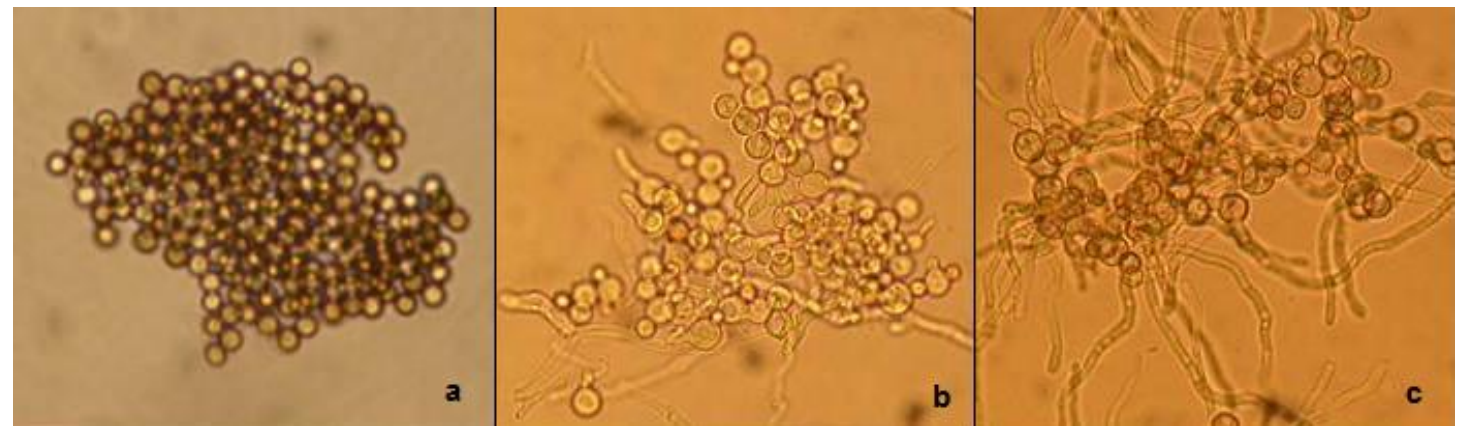

Fig 3

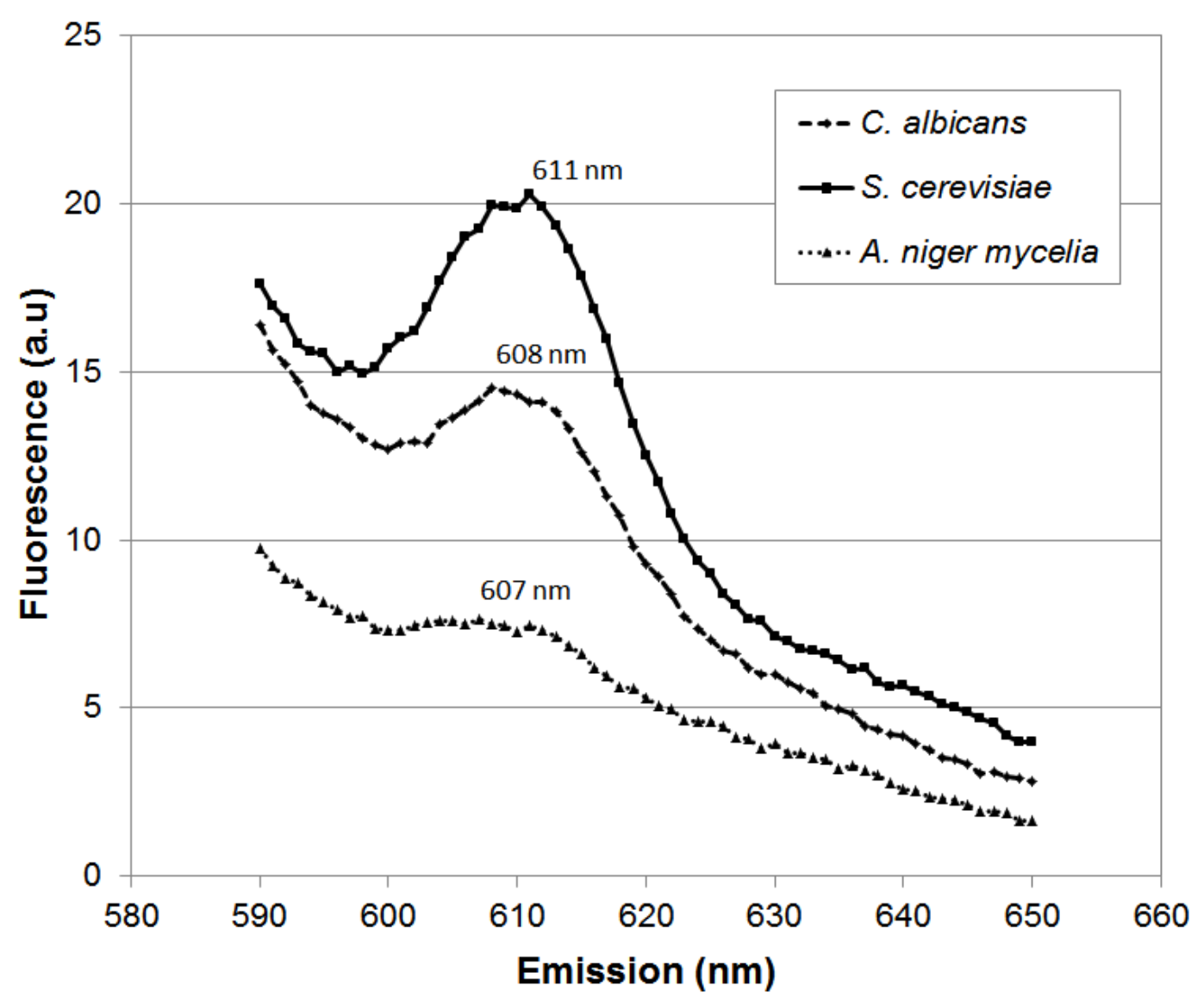

Fig 4 
783

784

785

786

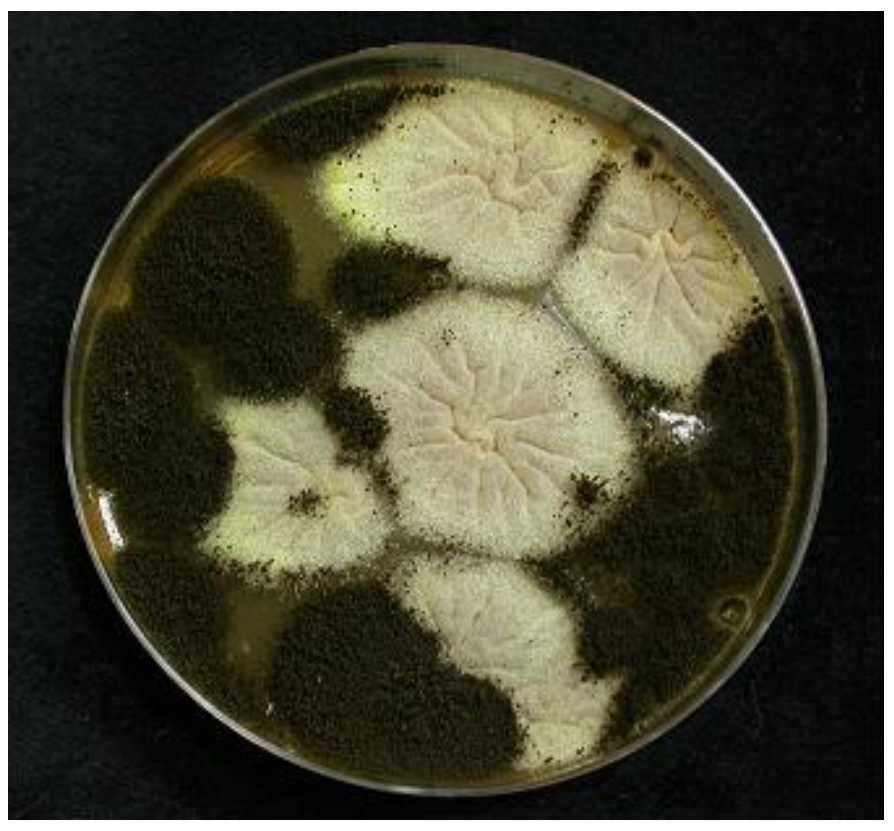

Fig 5

787

788 